\title{
Evolución de la gestión de la comunicación, empresas de energía eléctrica (Colombia)
}

\section{Communication management Evolution, electric power companies (Colombia)}

\author{
Dra. Graciela Tristancho Cediel (Colombia) \\ Corporación Universitaria Minuto de Dios \\ Doctorado en Comunicación Organizacional \\ gracielatristanch04@gmail.com
}

\section{Resumen}

En la última década la gestión de la Comunicación ha evolucionando favorablemente en el interior de las empresas colombianas del sector de la energía. Han pasado de ser solo oficinas de información, las cuales atendían a los medios de comunicación con notas de prensas, y/o a consumidores, proveedores, clientes, usuarios y demás público externo e interno. El presente trabajo pretende conocer las estrategias que han permitido la evolución de la relación con estos públicos, los cuales exigen cada día mayor atención y participación, y quienes requieren ser integrados en procesos de comunicación, con estrategias, diálogo, e investigación, para tipificarles, conocerles, y construir con ellos procesos de comunicación.

\section{Abstract}

In the last decade, communication management has favorably evolved within energy sector in Colombian companies. They have gone from being information offices only, which served media with press releases, and/or consumers, suppliers, customers, and external and internal public users. The present study aims to know the strategies that have allowed this relationship evolution, which demand every day more attention and participation, and require being integrated in communication processes, strategies, dialogue and research, to typify them, know them, and build communication processes.
Recibido:

Evaluado:

Aceptado:
17 de enero de 2017

4 de abril de 2017

15 de junio de 2017
PARA CITAR ESTE ARTÍCULO/TO CITE THIS ARTICLE

Tristancho Cediel, G. (2017). Evolución de la gestión de la comunicación, empresas de energía eléctrica (Colombia), Poliantea, 13(24), pág. 85-106. 
La presente es una investigación descriptiva de la evolución de la comunicación en tres empresas colombianas del sector de la energía en los última años; contiene un diagnóstico de la comunicación existente, y de las estrategias de comunicación utilizadas, las cuales han cobrado realce en la productividad y en los resultados de las compañías en mención, y en donde la comunicación se ha fortalecido, ampliado y consolidado. Como técnicas de recolección de datos, ralizadas entre el 2009 y 2010 , se complementó la información obtenida a través de la técnica de la observación directa, mediante la realización de una encuesta electrónica de opinión y entrevistas dirigidas a directores del área de comunicación, quienes en su momento solicitaron no mencionar sus nombres.

Palabras Clave: gestión de la comunicación, medios de comunicación, procesos de comunicación.
This is a descriptive investigation about communication evolution in three energy sector Colombian companies; offers both a current communication and communication strategies diagnosis, which have become relevant in productivity and in aforementioned companies results, and where communication has been strengthened, expanded and consolidated. As data collection techniques, made between 2009 and 2010, the information obtained were completed with direct observation, by conducting an online opinion survey and interviews addressed to communication area, whom requested anonymity.

Keywords: management of communication, communication media, communication processes. 


\section{Introducción}

La tendencia cambiante que adopta actualmente el mundo empresarial, permite que en el interior de este mundo se erijan importantes avances en la organización de la Comunicación, como regulador y optimizador del proceso de desarrollo de las empresas en general. La tendencia de cambio es global y obedece entre otras razones, a los incesantes e increíbles avances tecnológicos, al cambio climático, a la economía nacional e internacional, etc.

En nuestro país, el sector de la energía es un ejemplo de ello. Son organizaciones de vital importancia para la economía nacional, con sólida estructura financiera, eficientes redes comerciales, y con un comprometido crecimiento y desarrollo en la economía de Colombia. En su interior, se desenvuelven importantes avances en la organización de la comunicación, dignos de conocer a través de una investigación de tipo descriptiva, que nos permita conocer más de cerca el ejercicio actual de ésta profesión en empresas del sector eléctrico, y a su vez nos indique hasta qué punto la comunicación aplicada ha evolucionado integralmente, al incluir en sus estrategias a todos sus públicos, demostrando con ello, la madurez de ésta disciplina con resultados de productividad para la entidad y para el país en general. El estudio incluye un diag- nóstico general de la comunicación, e incluye además la revisión de elementos para una gestión óptima como la investigación, la evaluación del ejercicio de la Comunicación y la atención que se les brinda a los públicos implicados con esta organización.

De esta manera identificamos las buenas prácticas de comunicación introducidas en la cultura colombiana, y determinamos si la comunicación ha logrado en los últimos años evolucionar y posicionarse en el ámbito empresarial como una disciplina seria y estructurada para crear, y fortalecer el relacionamiento entre las organizaciones y sus públicos.

\section{Planteamiento del problema y justificación}

¿La Gestión de la Comunicación en empresas del sector eléctrico, ha evolucionado progresivamente en los últimos años, al incluir estrategias y evaluación de la comunicación con todos sus públi$\cos$ ?

\section{Objetivos}

\section{Objetivo general}

Analizar la evolución de la gestión estratégica de la comunicación con sus públicos de interés en empresas del sec- 
tor de la energía a nivel nacional, en el periodo 2005 - 2010.

\section{Objetivos específicos}

1. Realizar un diagnóstico de comunicación empresarial que sirva de herramienta de análisis y evaluación de la gestión en comunicación en la entidad como muestra participante.

2. Analizar la gestión actual de la comunicación en las organizaciones seleccionadas como muestra participante.

\section{Metodología}

En cuanto a la naturaleza del estudio, el presente trabajo adopta la forma de una investigación descriptiva, la cual como su nombre lo indica, se limita a describir el estado comunicacional en la empresas del sector mencionado, mediante los cuales se pretende especificar las características, el funcionamiento, y las estrategias empleadas en comunicación. Por esta razón, las carateristicas propias de la investigación realizada solo permiten obtener resultados conclusivos acerca del estado actual de la comunicación, pero no conducen a determinar las causas que lo originaron.

Técnicas de recolección de datos: Las principales técnicas de recolección de datos utilizadas fueron la observación directa, la encuesta y la entrevista.

Mediante la observación directa, se recopilaron datos relacionados con el estado físico en sí, así como de los elementos comunicacionales aplicados en cada empresa, con el fin de obtener informa- ción de primera mano que permitiera valorar su estado real, sin que la información que pudiera existir sobre él, en diversos medios de difusión, modificara o distorsionara su estado verdadero; la observación directa permitió:

1. Realizar un inventario de estrategias internas y externas

2. Mostrar las peculiaridades del entorno comunicacional

3. Identificar las diferencias comunicacionales entre las entidades visitadas

\section{Instrumentos de medición}

Se complementó la información obtenida a través de la observación, mediante la realización de una encuesta electrónica de opinión dirigida a los directores del área de comunicación.

Las preguntas del cuestionario de la encuesta y de la entrevista fueron verificados con los directores de comunicación de las empresas y redactadas de manera que se pudiera determinar cualitativa y cuantitativamente la siguiente información: (aplicadas al director y a su equipo de trabajo)

- Existencia de gestión de la comunicación en la empresa

- Actividades y funciones dentro del área de comunicación: Estrategias de comunicación interna y externa.

- Sistemas utilizados de evaluación de estrategias aplicadas en comunicación.

- Técnicas de investigación. 
- Cuantas personas componen el área de comunicación, sus profesiones y características del profesional que dirige el equipo.

Finalmente, se entrevistó a los directores del área de comunicación de cada empresa -quiénes solicitaron en su momento, no divulgar sus nombres- para complementar la información necesaria acerca de otros aspectos cualitativos importantes, como lo son, el diseño del organigrama del área o departamento de comunicación; detalles de la comunicación interna, de la comunicación externa, estrategias presentes y futuras para la comunicación. De manera específica se entrevistó a directores de las empresas: Ecopetrol; Emgesa; (ISA) Interconexión Eléctrica; (ESSA) Electrificadora de Santander; (EPM) Empresas Públicas de Medellín; Termotasajero.

\section{Marco Teórico}

Alejandro Alvarez Novel en su libro ?Medición y evaluación en Comunicación? señala que una de las caracteristicas de las relaciones públicas en las empresas de éxito es el hecho de medir realmente lo que piensa el público sobre las acciones comunicativas que se aplican, pues se mide las acciones de mercadeo, publicaciones en redes sociales y medios web y la atención en la evaluación del impacto, y muy especialmente, la modificación que se logró en el comportamiento, percepción y como habla el público al cual se dirigió cada una de las actividades comunicacionales ya sean internas y externas en las empresas.

El proceso histórico del estudio sobre la importancia de la medición de las comunicaciones en la comunicación había sido escaso hasta la década de 1950 cuando profesionales de las relaciones públicas descubrieron la importancia de medir la eficacia de sus acciones. Paulatinamente se ha incrementado el estudio del efecto de las comunicaciones en las personas y por esto la importancia de la medición y posterior evaluación de esta en las empresas, los padres de estos estudios fueron Harold D. Laswell, Paul F. Lazarsfeld y Carl Hovland en los que de evalúa el posible efecto psicosocial de las publicidad y las acciones comunicativas de las empresas y organizaciones.

El autor analiza el rol estratégico de la comunicación desde el punto de vista de las teorías de la Escuela clásica, la Escuela de Relaciones Humanas y la escuela sistémica de los que concluye que las relaciones públicas son un canal que relaciona a una empresa con sus públicos internos y externos en las que los relacionistas públicos dirigen, planifican, ejecutan y miden la comunicación, esta función puede ser aplicado en este campo desde diferentes modelos que son:

- Agentes de prensa/Publicity: Estos realizan la labor de propagación de información principalmente hacia los medios de comunicación.

- Información pública: Emite información pública sin ánimo de persuasión, solo informa abiertamente y completa para que el espectador tome la decisión.

- Asimétrico bidireccional: Busca la mejor manera de persuadir. 
- Simétrico bidireccional: Sirve de mediador entre las organizaciones y los públicos creando comunicaciones responsables sin buscar una dirección correcta para la razón incluyendo al cliente.

- Modelo bidireccional simétrico persuasivo: Es una mezcla de los dos últimos modelos, pero al final si busca persuadir.

\section{"Branding corportivo" escrito por Paul Capriotti en el año 2009}

Explica los fundamentos para la gestión estratégica de la identidad corporativa y la comunicación hacia las personas como su principal objetivo ya que la gran mayoría de organizaciones tienen problemas para identificar la identidad de su organización. En el libro Dentro del libro Brandig corporativo encontramos análisis y reflexiones sobre los principios, lineamentos y desarrollos dentro de una empresa las cuales son la base de identidad corporativa.

Los conceptos sobre la estructura y procesos corporativos están acompañados de las teorías de la comunicación, las relaciones públicas, la comunicación de la empresa y gestión empresarial. La información que se tiene al alcance en estos tiempos es mucho mas grande y valiosa de la que se tenia años atrás respecto a productos, servicios, actividades o empresas existentes, esto lo que hace es que no se tenga una fácil recordación de identidad, de reconocer las diferencias rápidas de unas y las otras; por esta razón es imperioso que las organizaciones construyan un proceso de gestión de identidad, desarrollen actividades que les ayude a posicionar una identidad que los identifique, y les permitan marcar la diferencia frente a las demás empresas, muy especialmente con su competencia directa.

Las organizaciones, los productos, las actividades, necesitan un identidad clara con características, y comunicación eficaz, con esto se logra que las personas identifiquen a la empresa y sus diferencias frente a las demás empresas existentes en el mercado, y generar más valor para que las prefieran frente a lo que exista en el mercado. Este resultado es conocido como imagen corporativa, son características o atributos que los individuos asocian a una organización. Este modelo de gestión es el BRANDING.

La identidad corporativa de una empresa tiene una influencia decisiva en todos los aspectos de gestión de una organización, pues esta orienta las decisiones, políticas, estratégicas y acciones de la empresa.Los factores que influyen en la identidad corporativa son muchos, pero algunos de ellos son los que pautan esta verdadera identidad, los más importantes son: La personalidad y normas del fundador; La personalidad $y$ normas de personas claves; La evolución histórica de la organización; La personalidad de los individuos; El entorno social. Por otro lado los componentes de la identidad corporativa son dos según la mayoría de autores consultados por Capriotti, por un lado tenemos EL ALMA, que la definen como aquello que la organización realmente es, y la segunda es LA MENTE, lo que la organización quiere ser. 
Es importante que dentro de una compañía existan canales de comunicación, de esta manera se pueden diferencias niveles de comunicación de una organización con sus públicos. Los medios masivos de comunicación y las relaciones interpersonales proveerán al individuo de forma socialmente mediada, mientras que la experiencia personal con las organizaciones que le aportará el sujeto la información directa experimentada. La identidad corporativa requiere de un trabajo estratégico, una visión total de la organización, para esto se debe trabajar en tres aspectos importantes: Un análisis estratégico de la situación actual de la compañía; Definir el perfil de identidad corporativa; La comunicación del perfil de la identidad corporativa.

\section{Relaciones Publicas y Comunicación Orga-} nizacional: Ejes de la comunicación "Guía de conceptos" Gloria Consuelo Fajardo Valencia y Adriana Marcela Nivia. 2016

Relaciones Públicas y comunicación organizacional, cuenta diferentes experiencias en comunicación organizacional en donde las relaciones públicas han favorecido el crecimiento de las empresas, y por ende el aumento de su rentabilidad. Las relaciones públicas sigue conquistando nuevos espacios en diferentes escenarios, con procesos de calidad.

\section{Evolución de las Relaciones Púbicas}

Las relaciones públicas han tenido un cambio y trasformación en el mundo organizacional, en donde la comunicación en ocasiones, ha sido reemplazada por otras áreas profesionales no idóneas para el cargo. Desde el origen del hombre, siempre ha existido la necesidad de comunicarse de diferentes formas, la palabra ha representado el mejor medio expresivo en los diferentes escenarios políticos, religiosos, sociales y culturales. Con el paso del tiempo sigue evolucionado con nuevas herramientas para tener más interacción. En las crisis mundiales los periodistas aprovecharon para hacer relaciones públicas; en la segunda guerra mundial se produjo un crecimiento en todas las áreas de relaciones públicas, en siglo XX se afianza la profesión, las empresas abrieron departamentos de relaciones públicas y ampliaron los existentes, a finales del mismo siglo los empresarios entendieron gracias los conflictos sociales de la época, lo fundamental de gozar de uan excelente reputación, se crearon entonces nuevas técnicas de comunicación, con estrategias dentro de la propia crisis. Las relaciones públicas se desarrollaron de acuerdo al entorno social.

\section{América, Europa y Colombia}

Primer Modelo: Agente de prensa, en el modelo de las relaciones públicas, es la persona que logra Publicity, su función es emitir comunicados y avisos de prensa sobre las organizaciones o individuos. "Julio Cesar y Henry Ford, Tom Thumb y P.T.Barnum"

Segundo Modelo: Lo propuso "lvy L Lee (1877-1934)", un asesor debe negociar con directivos no puede hacer pronunciamientos hasta que no tenga el apoyo de todos para dar información pública. En Europa las relaciones publicas evolucionó y se ligó a la política de esa época. 
En Alemania durante la revolución industrial, surgió la necesidad de informar al público, de de lo que estaba sucediendo en las empresas con las operaciones de las fábricas. Las industrias se consolidaron con el tiempo.

Colombia presenta un desarrollo tardío en comparación con EEUU y Europa. Sobre los años setenta llego el auge de las comunicaciones. Las relaciones públicas no tenían la misma importancia que en Estados Unidos, se consideraba que los profesionales de comunicación carecían de conocimientos integrales; el manejo de públicos era una labor poco importante, cualquier otro profesional podía desarrollar su papel; el ambiente empresarial solo tenía un aspecto social (almuerzos, cocteles, cenas), lo realizaban los abogados, cancilleres, diplomáticos, y quienes hacían labor de comunicación.

En 1994 evolucionó el concepto de Relaciones Públicas con patrocinios y eventos como herramientas poderosas, dentro de las jerarquías empresariales, sin abarcar clientes internos y externos. Su gran desarrollo se dío en 1998 cuando las empresas se dan cuenta de la importancia de la comunicación con los medios directos e indirectos, manejo de crisis, y de la buena imagen corporativa. Hoy se tiene un desafío de crecimiento para América Latina y Colombia, acorde al crecimiento económico y a la privatización de las empresa.
Los Cuatro Modelos de Comunicación en las Relaciones Públicas

Los catedráticos James Gruning, de la universidad de Maryland, y Todd Hunt,de Rutgers, dela universidad estatal de NuevaJersey ${ }^{1}$, elaboraron $\mathrm{cu}^{-}$ atro modelos de relaciones públicas: Agente de prensa; Información pública; Asimétrico bidireccional; Simétrico bidireccional; son modelos que nos ayudan a comprender la historia las Relaciones Públicas, disciplina que integra la Comunicación en las relaciones con todos los públicos.

En todas las empresas donde hay función de la comunicación se aplican alguno de estos modelos, pero cada uno de ellos nos indica hasta qué punto la comunicación aplicada es integral e incluye a todos sus públicos, y permitiendo la evolución y madurez del ejercicio de la Comunicación, con mayores resultados de productividad para las empresas y la sociedad en general. Los cuatro se practican en la actualidad, y cada modelo puede ser funcional para diferentes clases de organizaciones, dependiendo de los entornos y circunstancias, aunque de manera imperfecta o incompleta, salvo el modelo simétrico bidireccional, veamos porqué:

\section{Publicity/Agente de Prensa}

Los deportes, el teatro y la promoción de productos son los principales sectores que practica este modelo en la actualidad. La propaganda es el objetivo, a través de una comunicación de sentido único o unidireccional que, amenudo, es

\footnotetext{
${ }^{1}$ GRUNIG, James E, ToddHunt. “Dirección de Relaciones Públicas”. Barcelona: Gestión 2000-2003. Página 10
} 
incompleta, distorsionada, o solo cierta a medias. El modelo es del tipo fuentereceptor. La comunicación es considerada como una cuestión de contar, no de escuchar, investigándose poco, si es que se llega a investigar. P.T Barnum fue la principal figura histórica durante la vigencia de este modelo, desde 1850 hasta 1900.

\section{Información Pública}

Los gobiernos, las organizaciones sinánimo de lucro y algunas empresas son las principales instituciones que utilizan este modelo en la actualidad. La divulgación de información, sin que necesariamente tenga una intención de persuasión, es el principal objetivo. El modelo es de tipo fuente-receptor.Si se lleva a cabo algún tipo de investigación, probablemente quede confinada a simples tests oestudios de audiencia. IvyLee es la principal figura histórica de este modelo que empezó a desarrollarse entre1900y 1920.

\section{Asimétrico Bidireccional}

Las empresas competitivas y de relaciones públicas son las que más lo utilizan. La persuasión científica es el objetivo, y la comunicación se hace en doble sentido, con efectos distintos. El modelo es fuente-receptor, con retroalimentación de la fuente. La información es al tiempo formativo, ayudando a planificar una actividad y a elegir los objetivos, evalúa resultados para saber si ha alcanzado objetivos. EdwardL-Bernays es la principal figura histórica de este modelo, que empezó a utilizarse en la década de los años veinte.

\section{Simétrico Bidireccional}

Muy pocas empresas hasta el momento logran llegar a este modelo óptimo. El objetivo consiste en lograr una comprensión mutua, empresa y públicos, y la comunicación se produce en doble sentido con efectos análogos. El modelo es del tipo grupo-grupo, con retroalimentación. La investigación formativas e utiliza principalmente para conocer sobre la percepción del público respecto a la organización,y para determinar cuáles son los efectos de la organización sobre él público, permitiendo así asesorar a los directivos sobre las políticas a aplicar o directrices a seguir.

Las prácticas simétricas como asimétricas forman parte de unas Relaciones Publicas excelentes. Sin embargo puede presentarse, que los profesionales que utilizan las prácticas asimétricas persuasivas lo hacen no solo para persuadir a los públicos para que hagan lo que las organizaciones quieren que hagan.También persuaden a la Dirección para que cambie los comportamientos que tienen un efecto negativo en los públicos.

\section{Relaciones Públicas Optimas}

Rafael Pérez en su libro "Estrategias de comunicación" " nos enseña que lograr la optimización en la función de la Comunicación, requiere que los profesionales de ésta profesión participen en la dirección estratégica general de la or-

${ }^{2}$ PEREZ, Rafael. Estrategias de Comunicación.2001. P-472 
ganización, además de atender eficientemente todos los públicos de una organización, tanto internos como externos. Estrategias de comunicación basados en la teoría de investigaciones, la cual permiten tipificarlos públicos. "La estrategia acota un camino, y facilita un esquema al director, permite canalizar el poder de la comunicación, liderar ideas, coordinar equipos,concentrar esfuerzos".

Igualmente señala el autor que las estrategias de evaluación son imprescindibles en la actividad de la comunicación, para medir toda acción implementada por la Dirección; es esencial evaluar la aceptación y comprensión del mensaje por parte de los públicos y confirmar si responden a los objetivos de la organización. Se evalúa por fases: Especificación de objetivos, medición de objetivos, recogida y análisis de datos, comunicar resultados a quien toma las decisiones. Método de evaluación: Encuestas, entrevistas personales; entrevistas telefónicas; Cuestionarios por correo; experimentos, trabajo de campo.

En general las decisiones estratégicas tienen importancia para todos los públicos implicados con la organización, tanto internos como externos. Según D.Wilcox ${ }^{3}$ en el libro Relaciones Públicas. Estrategias y tácticas, define estos públicos integralmente de la siguiente manera:

Relaciones Públicas Externas: Les ofrece atención a los públicos externos. Asesora a asociaciones implicadas con la empresa. Se comunica con los accionistas del sector, a clientes o proveedores; a la comunidad en general. Marketing apoya esta actividad con la publicidad y sus diferentes manifestaciones incluyendo el patrocinio.

Relaciones Públicas Internas: Gestiona los públicos internos tanto empleados como directivos. Se relaciona con recursos humanos, juntos gestionan el comportamiento del trabajador para engranarlo al objetivo de la compañía.

Relaciones Públicas y Medios de Comunicación: Relación con periodistas representantes de responsabilidad ante los Comunicados de Prensa, Conferencias, Reportajes, Dossier.

Asuntos Públicos: Comunicación Institucional con los partidos políticos y organizaciones públicas, sindicatos. "Grupos de presión (LOBY) o colectivo que tiene un interés común, y toma medidas para defender sus intereses".

La Situación de crisis: Relaciones Publicas con los medios, los periodistas transmiten información "urgente" en época de crisis por medio de desayunos, reuniones, contacto permanente con equipo interno y externo; acuerdos redacción tipo informativo.

Si las organizaciones tienen buenas relaciones con los públicos, estas a su vez generan una reputación entre estos públicos. Por lo tanto los profesionales de la Comunicación gestionan la reputación de la Institución, al participar en la dirección estratégica de la

\footnotetext{
${ }^{3}$ D, Wilcox. Relaciones Públicas. Estrategias y tácticas. 2000. P-10
} 
organización, en la identificación de públicos estratégicos,y en la utilización de programas de comunicación para crear unas relaciones perdurables a largo plazo con estos públicos.

Precisa Joan Costa ${ }^{4}$ "la imagen es la resultante de múltiples y diversos mensajes de la empresa, relacionados entre sí, que acumulados en la memoria colectiva, configuran una globalidad significativa capaz de influir en los comportamientos y determinarlos. La Imagen Corporativa es,la expresión más concreta y visual de la identidad de una empresa, organismo o institución. En un mercado tan competitivo y cambiante, la imagen es un elemento definitivo de diferenciación y posicionamiento".

\section{Evolución de la comunicación en Colombia (Sector eléctrico)}

\section{Ecopetrol}

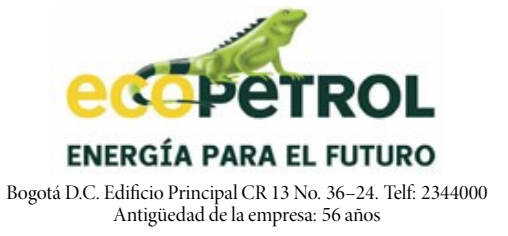

Denominación función de comunicación COMUNICACIÓN EXTERNA Y ÁREA ORGANIZACIONAL

\section{COMUNICACIÓN EXTERNA:}

Dependencia Jerárquica: PRESIDENCIA Antigüedad: 20 AÑOS

Personas que lo componen: 15 PERSONAS

ÁREA ORGANIZACIONAL:

Dependencia Jerárquica: Dirección de desarrollo Antigüedad: 12 ANOS

Personas que lo componen: 15 PERSONAS
Comunicación externa y área organizacional

La importancia del tema de la Comunicación en la empresa ECOPETROL, ha estado supeditada tanto a las políticas del Presidente de la compañía, como a las del Gobierno. Hacia 1996 se realizó un proceso de comunicación organizacional muy maduro y fortalecido que incluso llegó a ser modelo a nivel de distintas empresas en Colombia. Los procesos atendían a las distintas necesidades de la organización tendientes a informar no sólo a los trabajadores, sino a sus familiares, incluso a los hijos. Se disponía de distintos medios estructurados para cada público, con planes anuales trabajados sobre encuestas de necesidades. Para ese entonces se contaba igualmente con un grupo de profesionales en cada área operativa (campos de producción, transporte y refinación) que facilitaban la comunicación organizacional.

Entre 1998-1999 el precio del petróleo cae y la empresa se ve en la obligación de reducir su nómina, entran igualmente decretos de austeridad que afectan en primer lugar los presupuestos de las áreas de comunicaciones, se limitan las publicaciones y la producción de medios, entre otras actividades dirigidas a los trabajadores. En los años 2000-2002 el precio del petróleo vuelve a subir, hay una recuperación en la economía del país y a nivel de Gobiernos se suaviza un poco el tema. En ese sentido se trata de fortalecer los medios que no fueron eliminados por los decretos de austeridad. El Presidente de ese entonces, fortalece el tema

\footnotetext{
${ }^{4}$ COSTA, Joan, Imagen Corporativa en el siglo XXI, Ed. La Crujía ediciones. 1999.
} 
de la Comunicación Directa y se trabaja sobre bases de mantener información permanente dentro de la organización, dando respuesta a sus inquietudes. También se comienza el tema de la intranet, aprovechando el acceso a computadores de una buena parte del personal y a la agilidad que el tema da.

Del año 2002 a la fecha hay cambio de Gobierno así como de políticas. La empresa fue prescindida de su papel histórico de administrador del recurso hidrocarburo, lo que conlleva también cambios profundos en su estructura organizacional. Hoy se trabaja sobre el fortalecimiento del papel que juega las comunicaciones organizacionales en la nueva compañía y en ese sentido se replanteó el tema de las comunicación es a través de los procesos de comunicación directa, informativa y comunicación virtual, se trabaja sobre planes anuales, atravesándola organización con mensajes corporativas y puntualizando las diferencias para cada área operativa (campos de producción, estaciones de transporte, refinerías).

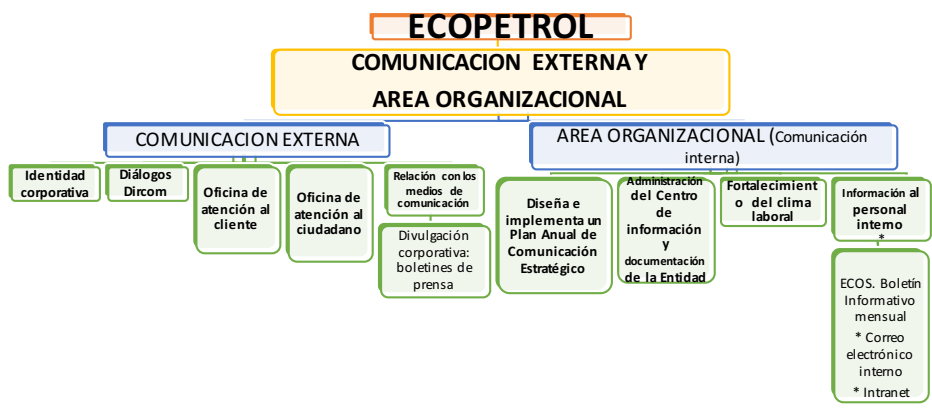

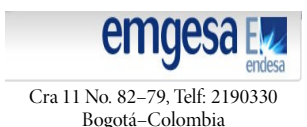

\section{Gerencia de comunicación EMGESA}

Denominación función de Comunicación: GERENCIA DE COMUNICACIONES Antigüedad dto Comunicación: 15

Dependencia jerárquica: GERENCIA GENERAL

Antigüedad empresa: 18 años

Antigüedad dto. Comunicación: 15 años Personas que lo componen: 10 PERSONAS
La Gerencia se crea en 1999 y se denominó Asuntos Corporativos, la gestión de esta área se concentraba principalmente en encauzar sus esfuerzos dentro de una estrategia de participación y autogestión con las comunidades y autoridades locales y regionales.

Los temas comunicación externa se desarrollaban con el fin de prevenir y controlar los efectos relacionados con la falta de información y expectativas de las comunidades y autoridades municipales, acerca de los aspectos impor- 
tantes de la operación. Adicionalmente se celaba por consolidar la imagen corporativa en las áreas de influencia.

En el 2005, la Gerencia toma un rumbo diferente, afianzando y fortaleciendo las áreas de comunicación interna y externa, relacionamiento corporativo e institucional y gestión social, convirtiéndose en un área estratégica de la Compañía.

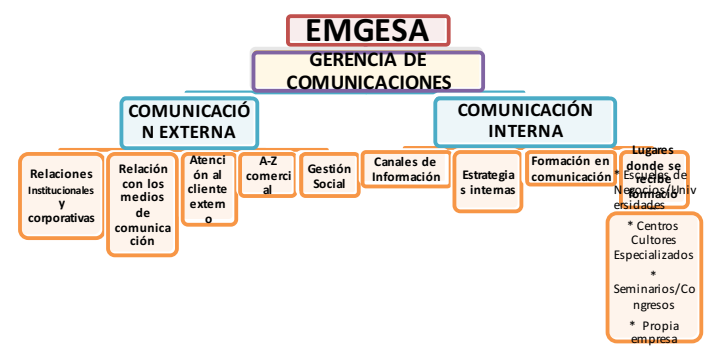

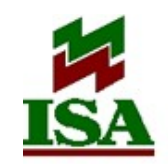

Calle 12 sur No. 18-168, Telf: (57)43252270 Medellín Colombia

\section{ISA (Interconexión eléctrica)}

Denominación función de Comunicación: PLANEACIÓN CORPORATIVA

Dependencia jerárquica:

GERENCIA GENERAL

Antigüedad empresa: 40 años

Antigüedad dto. Comunicación: 20

Personas que lo componen: 12

\section{Planeación Corporativa}

Con respecto a las comunicación, ISA tiene una trayectoria de casi 30 años, en los cuales se ha evidenciado un proceso de transformación que se originó en el desarrollo de acciones de comunicación interna y prensa y ha ido evolucionando, consecuente con el desarrollo conceptual de las comunicaciones en el campo internacional, hasta adoptar un modelo de comunicación corporativa que tiene una mirada más estratégica y se extiende aun mercado y unos grupos de interés mucho más amplios, con los cuales hemos venido construyendo procesos de relacionamiento. Hoy cuentan con un enfoque hacia el gerenciamiento de la marca, desarrollado bajo valiosas herramientas de gestión como las políticas de comunicación e información, la guía de gerenciamiento de marca y la gestión integral de riesgos.

El direccionamiento estratégico de la compañía se ve traducido en una estrategia de posicionamiento que busca mantener la imagen de ISA entre sus diferentes públicos, siendo consistentes con los atributos de marca que presentan una empresa sólida, rentable,seria,confiable y con proyección futura, líder en operación, administración y transporte de mercados de energía en Colombia y Latinoamérica, 
para lo cual se deben seleccionar los medios más afines para los diferentes segmentos.

Existen en ISA funciones de comunicación corporativa para el Grupo ISA y para ISA,empresa matriz, que están a cargo de la Dirección Proyección Cor- porativa. Pero adicionalmente, desarrollamos actividades de comunicación para el desarrollo, a cargo de la Dirección Social y Ambiental, en coordinación con la Dirección Proyección Corporativa. Para efectos de este documento el énfasis estará en los procesos de Proyección Corporativa.

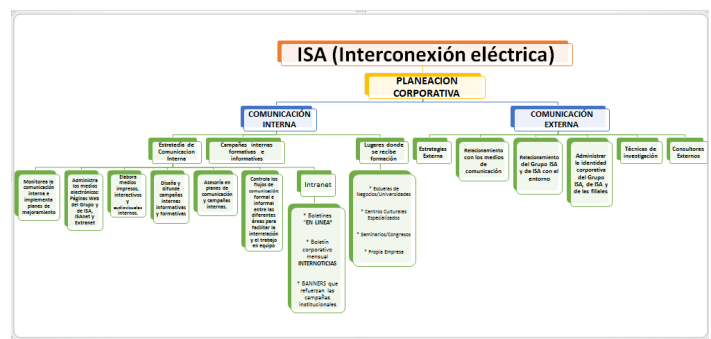

\section{Tablas y Gráficos}

\begin{tabular}{|l|c|c|}
\hline \multicolumn{3}{|l|}{ Número de personas integran dpto comunicación } \\
\hline Item & Cantidad & Porcentaje \\
\hline 1 a 10 personas & 5 & $71 \%$ \\
\hline 11 a 20 personas & 2 & $29 \%$ \\
\hline 21 a 30 personas & 0 & $0 \%$ \\
\hline Total & 7 & $100 \%$ \\
\hline
\end{tabular}

\begin{tabular}{|l|c|c|}
\hline \multicolumn{3}{|c|}{ Dependencia jerárquica del Depto de Comunicación } \\
\hline Item & Cantidad & Porcentaje \\
\hline Comunicación & 3 & $43 \%$ \\
\hline Comunicación corporativa & 2 & $29 \%$ \\
\hline Comunicación interna & 1 & $14 \%$ \\
\hline Comunicación externa & 0 & $0 \%$ \\
\hline $\begin{array}{l}\text { Comunicación interna y relaciones } \\
\text { externas }\end{array}$ & 1 & $14 \%$ \\
\hline Dirección de desarrollo & 0 & $0 \%$ \\
\hline Total & 7 & $100 \%$ \\
\hline
\end{tabular}
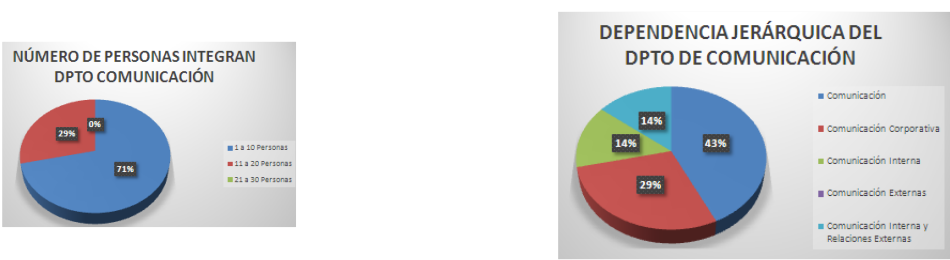

\begin{tabular}{|l|c|c|}
\hline \multicolumn{3}{|c|}{ Formación persona responsable comunicación } \\
\hline Item & Cantidad & Porcentaje \\
\hline $\begin{array}{l}\text { Pregrado en Comunicación So- } \\
\text { cial }\end{array}$ & 1 & $14 \%$ \\
\hline $\begin{array}{l}\text { Pregrado con Especialización } \\
\text { en Comunicación }\end{array}$ & 3 & $43 \%$ \\
\hline $\begin{array}{l}\text { Pregrado con Especialización y } \\
\text { Master }\end{array}$ & 2 & $29 \%$ \\
\hline $\begin{array}{l}\text { Profesional en Otras Áreas con } \\
\text { Especialización }\end{array}$ & 1 & $14 \%$ \\
\hline Total & 7 & $100 \%$ \\
\hline
\end{tabular}

\begin{tabular}{|l|c|c|}
\hline \multicolumn{3}{|l|}{ Antigüedad del departamento de comunicación } \\
\hline Item & Cantidad & Porcentaje \\
\hline 1 a 10 años & 1 & $14 \%$ \\
\hline 11 a 20 años & 6 & $86 \%$ \\
\hline 21 a 30 años & 0 & $0 \%$ \\
\hline Total & 7 & $100 \%$ \\
\hline
\end{tabular}
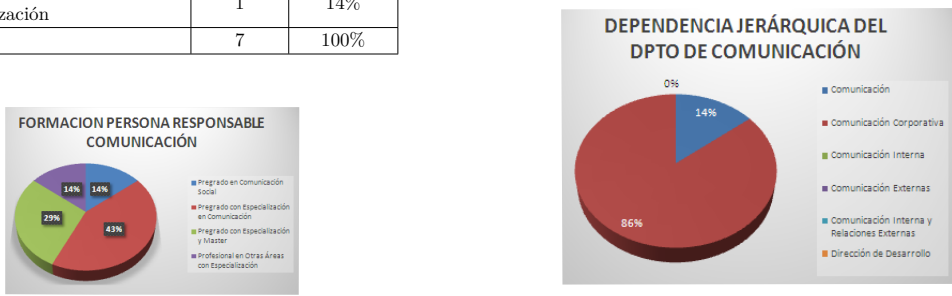


\begin{tabular}{|l|c|c|}
\hline \multicolumn{3}{|c|}{ Formación persona responsable comunicación } \\
\hline Item & Cantidad & Porcentaje \\
\hline $\begin{array}{l}\text { Pregrado en Comunicación So- } \\
\text { cial }\end{array}$ & 1 & $14 \%$ \\
\hline $\begin{array}{l}\text { Pregrado con Especialización en } \\
\text { Comunicación }\end{array}$ & 3 & $43 \%$ \\
\hline $\begin{array}{l}\text { Pregrado con Especialización y } \\
\text { Master }\end{array}$ & 2 & $29 \%$ \\
\hline $\begin{array}{l}\text { Profesional en Otras Áreas con } \\
\text { Especialización }\end{array}$ & 1 & $14 \%$ \\
\hline Total & 7 & $100 \%$ \\
\hline
\end{tabular}

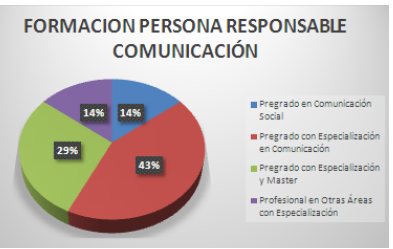

\begin{tabular}{|l|c|c|}
\hline \multicolumn{3}{|l|}{ Número de personas integran dpto Comunicación } \\
\hline Item & Cantidad & Porcentaje \\
\hline 1 a 10 Personas & 5 & $71 \%$ \\
\hline 11 a 20 Personas & 2 & $29 \%$ \\
\hline 21 a 30 Personas & 0 & $0 \%$ \\
\hline Total & 7 & $100 \%$ \\
\hline
\end{tabular}

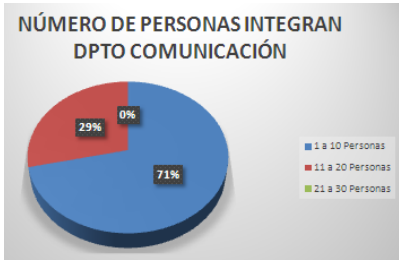

\begin{tabular}{|l|c|c|}
\hline \multicolumn{3}{|c|}{ Formación del personal } \\
\hline Item & Cantidad & Porcentaje \\
\hline $\begin{array}{l}\text { Pregrado en Comunicación So- } \\
\text { cial }\end{array}$ & 2 & $29 \%$ \\
\hline $\begin{array}{l}\text { Pregrado / profesional en co- } \\
\text { municación con especialización }\end{array}$ & 1 & $14 \%$ \\
\hline $\begin{array}{l}\text { Becario pregrado / profesional } \\
\text { en comunicación }\end{array}$ & 0 & $0 \%$ \\
\hline $\begin{array}{l}\text { Técnico en comunicación in- } \\
\text { terna }\end{array}$ & 1 & $14 \%$ \\
\hline Auxiliar de comunicación & 1 & $14 \%$ \\
\hline $\begin{array}{l}\text { Pregrado / profesional en otras } \\
\text { áreas }\end{array}$ & 1 & $14 \%$ \\
\hline Técnico en otras profesiones & 1 & $14 \%$ \\
\hline Total & 7 & $100 \%$ \\
\hline
\end{tabular}

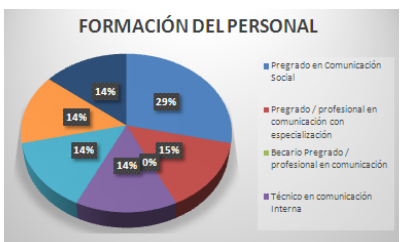

\begin{tabular}{|l|c|c|c|}
\hline \multicolumn{4}{|c|}{ Estrategias de comunicación } \\
\hline Item Cantidad & Porcentaje \\
\hline $\begin{array}{l}\text { Mercadeo / imagen corporativa } \\
\text { y publicitaria }\end{array}$ & 7 & $58 \%$ \\
\hline $\begin{array}{l}\text { Comunicación interna y ex- } \\
\text { terna }\end{array}$ & 2 & $17 \%$ \\
\hline $\begin{array}{l}\text { Planeación estratégica y fi- } \\
\text { nanciera }\end{array}$ & 2 & $17 \%$ \\
\hline No existen & 1 & $8 \%$ \\
\hline Total & 12 & $100 \%$ \\
\hline
\end{tabular}

ESTRATEGIAS DE COMUNICACIÓN

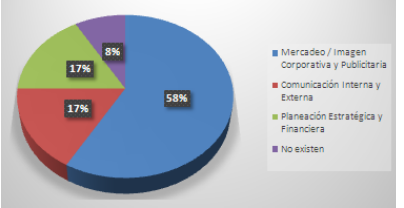

\begin{tabular}{|l|c|c|}
\hline \multicolumn{3}{|c|}{ Estrategias de comunicación } \\
\hline Item & Cantidad & Porcentaje \\
\hline $\begin{array}{l}\text { Planeación estratégica en co- } \\
\text { municación interna y externa }\end{array}$ & 6 & $40 \%$ \\
\hline $\begin{array}{l}\text { Mercadeo / imagen corporativa } \\
\text { y publicitaria }\end{array}$ & 3 & $20 \%$ \\
\hline No existen & 6 & $40 \%$ \\
\hline Total & 15 & $100 \%$ \\
\hline
\end{tabular}

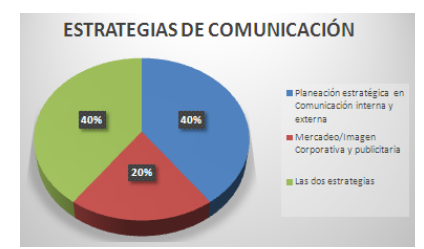

\begin{tabular}{|l|c|c|}
\hline \multicolumn{3}{|c|}{ Dependencia comunicación interna } \\
\hline Item & Cantidad & Porcentaje \\
\hline Dirección de comunicación & 5 & $71 \%$ \\
\hline No existen & 2 & $29 \%$ \\
\hline Total & 7 & $100 \%$ \\
\hline
\end{tabular}

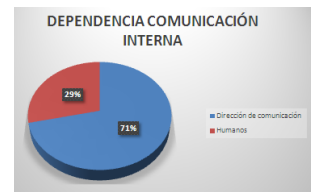

\begin{tabular}{|l|c|c|}
\hline \multicolumn{3}{|l|}{ ¿Utilizan técnicas de investigación? } \\
\hline Item & Cantidad & Porcentaje \\
\hline $\mathrm{Si}$ & 5 & $71 \%$ \\
\hline No & 2 & $29 \%$ \\
\hline Total & 7 & $100 \%$ \\
\hline
\end{tabular}




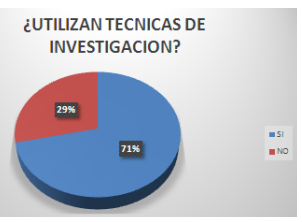

\begin{tabular}{|l|c|c|}
\hline \multicolumn{3}{|c|}{ ¿Cúales técnicas de investigación? } \\
\hline Item & Cantidad & Porcentaje \\
\hline $\begin{array}{l}\text { Cualitativo comunicación in- } \\
\text { terna }\end{array}$ & 2 & $29 \%$ \\
\hline $\begin{array}{l}\text { Cualitativo comunicación ex- } \\
\text { terna }\end{array}$ & 1 & $14 \%$ \\
\hline $\begin{array}{l}\text { Comunicación interna (sondeos } \\
\text { de opinión al personal) }\end{array}$ & 2 & $29 \%$ \\
\hline $\begin{array}{l}\text { Relaciones públicas (sondeos } \\
\text { de opinión al público externo) }\end{array}$ & 2 & $29 \%$ \\
\hline Total & 7 & $100 \%$ \\
\hline
\end{tabular}

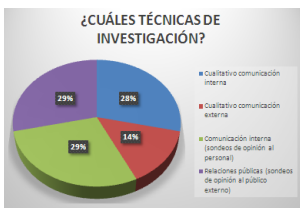

\begin{tabular}{|l|c|c|}
\hline \multicolumn{3}{|c|}{ Técnicas para evaluar la comunicación } \\
\hline Item & Cantidad & Porcentaje \\
\hline $\begin{array}{l}\text { Evaluación y aceptación del } \\
\text { mensaje }\end{array}$ & 1 & $14 \%$ \\
\hline $\begin{array}{l}\text { Evaluación de políticas imple- } \\
\text { mentadas }\end{array}$ & 2 & $29 \%$ \\
\hline Autoría de relaciones humanas & 2 & $29 \%$ \\
\hline Satisfacción de la comunicación & 2 & $29 \%$ \\
\hline Total & 7 & $100 \%$ \\
\hline
\end{tabular}
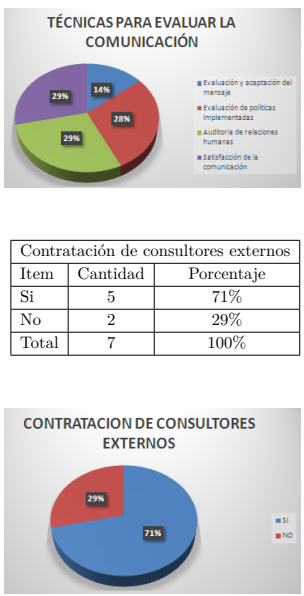

\begin{tabular}{|l|c|c|}
\hline \multicolumn{3}{|c|}{ Contratación de consultores externos } \\
\hline Item & Cantidad & Porcentaje \\
\hline Si & 5 & $71 \%$ \\
\hline No & 2 & $29 \%$ \\
\hline Total & 7 & $100 \%$ \\
\hline
\end{tabular}

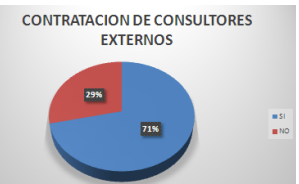

\begin{tabular}{|l|c|c|}
\hline \multicolumn{2}{|l|}{ Evaluación de la dirección y gestión de la comunicación } \\
\hline Item & Cantidad & Porcentaje \\
\hline Si evalúa & 5 & $71 \%$ \\
\hline No evalúa & 1 & $14 \%$ \\
\hline No siempre & 1 & 14 \\
\hline Total & 7 & $100 \%$ \\
\hline
\end{tabular}

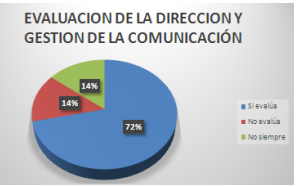

\begin{tabular}{|l|c|c|}
\hline \multicolumn{3}{|c|}{ Dependencia jerárquica del dpto de comunicación } \\
\hline Item & Cantidad & Porcentaje \\
\hline $\begin{array}{l}\text { Administración gerencia gen- } \\
\text { eral, Presidente, Secretaria } \\
\text { general }\end{array}$ & 7 & $88 \%$ \\
\hline Otras áreas & 1 & $12 \%$ \\
\hline Total & 8 & $100 \%$ \\
\hline
\end{tabular}

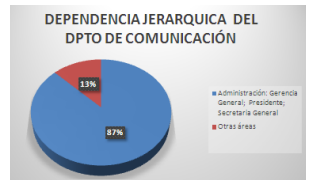

\begin{tabular}{|l|c|c|}
\hline \multicolumn{3}{|c|}{ ¿Comunicación interna depende de recursos humanos de comunicación? } \\
\hline Item & Cantidad & Porcentaje \\
\hline Dirección de comunicación & 6 & $86 \%$ \\
\hline Recursos humanos & 1 & $14 \%$ \\
\hline Total & 7 & $100 \%$ \\
\hline
\end{tabular}

¿COMUNICACIÓN INTERNA DEPENDE DE RECURSOS HUMANOSO DECOMUNICACIÓN?

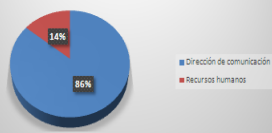

\section{Conclusiones}

Los Departamentos de Comunicación han experimentado en la última década crecimiento y evolución, permanencia en el medio, y caracterización de 
acuerdo a los modelos de las Relaciones Públicas, los cuales contienen principios genéricos que funcionan en todo el mundo, aunque aplicados de forma diferente, y específica en cada empresa en mención, de acuerdo a las condiciones culturales, políticas, económicas, y niveles de desarrollo. La gestión de la comunicación ha pasado paulatinamente de ser una simple relación de promoción y relación con los medios a ser una función de Gestión y Dirección con todos los públicos implicados con la organización, confirmando la hipótesis planteada inicialmente.

En esta última década del ejercicio de la profesión, necesariamente son muchas las modificaciones que se han tenido que realizar, en las actitudes y pautas profesionales iniciales, pero actualmente han finalizado su fase de implantación del área de Comunicación dentro de las empresas, y ahora su necesidad constante es la de permanecer en el mercado a nivel nacional e internacional. La Comunicación inició su función desde hace 15 ó 20 años, en cada una de ellas, como oficinas de comunicación, cuyo objetivo primordial era el de realizar boletines de prensa para los medios de comunicación y/o informar a sus públicos más cercanos. Hoy en día la gestión ha evolucionado hacia la investigación, estrategias, evaluación y gestión con todos sus públicos.

Dentro de los Departamentos de comunicación, se guarda similitud en la gestión, en la atención a sus públicos: comunicación interna, externa, con los medios de comunicación, con asuntos públicos. Existen algunas diferencias no muy significativas dentro de la función, como por ejemplo, la denominación de los nombres para designar la actividad de la comunicación.

Estrategias de comunicación: A medida en que las estrategias de comunicación han cobrado realce en la productividad y en los resultados de estas compañías, estos departamentos se han fortalecido y ampliado, aunque también son de los más vulnerables a las crisis financieras y presupuestales, por considerárseles susceptibles de privatizaciones, como es el caso de ECOPETROL, CORELCA.

Tienen estrategias expresadas bajo nombres diferentes; estrategias corporativas: estrategia de campaña, de comunicación interna, de comunicación externa, de marketing, creativa, de medios, del mensaje, promociónales, de imagen, de marca, de públicos, de programación. Estrategia de consultores externos: para la valoración de sus funciones; para analizar de forma independiente y externa situaciones y problemáticas de sector y temas internos; para actualizar y renovar políticas comunicación. Estrategias ante situaciones de crisis: es la posición de la empresa frente al momento álgido, y frente a las decisiones tomadas. Las crisis afectan directamente la imagen corporativa.

Las empresas estudiadas del sector energético, tienen una estructura comunicacional dividida en dos ramas, las comunicaciones internas y las comunicaciones externas, aunque estas últimas son, por lo general, de más antigua creación. 
Comunicación externa: busca atender las peticiones de información requeridas por los distintos públicos externos a la entidad y, por otro lado, informar por iniciativa propia sobre aquellos temas que se consideran relevantes en el desarrollo de la actividad. Las formas más habituales de comunicación con los organismos externos son los comunicados de prensa, los portales de Internet, las ruedas de prensa, las entrevistas con voceros autorizados o las declaraciones institucionales, los informes especiales (dossier), los recorridos de medios, la documentación textual y visual, las revistas y los video-clips institucionales, así como la organización de eventos, la cobertura de congresos y otros actos similares.

Comunicación interna: es relevante, hoy en día, dentro de estos Departamentos, el progreso sistemático de la $\mathrm{Co}$ municación Interna, como herramienta de suma importancia para los resultados empresariales. Los programas simétricos enfatizan la comunicación interpersonal y el dialogo con la dirección, e incluyen sesiones de información a los empleados y seminarios por circuito cerrado, cintas de video y formación en comunicación interpersonal entre directivos. En todas las organizaciones vistas, se construye una relación próxima con los empleados dentro de los programas existentes, los cuales van más allá de la simple acción técnica o de producción de un periódico, o revista. La manera que los trabajadores perciben a la alta dirección influye en la satisfacción del trabajo más que cualquier otro aspecto. Los trabajadores quieren trabajar para organizaciones que tienen un CLIMA organizacional que les genere confianza, y es- tabilidad.

Anteriormente, en empresas como Gecelca, (antiguamente Corelca), la comunicación interna dependía de la dirección de recursos humanos de la entidad. Actualmente en las empresas del sector eléctrico, por estrategia, la Comunicación Interna depende directamente del departamento de comunicación, o cualquiera que sea su denominación. Recursos humanos se dedica a la contratación y al bienestar general del trabajador, y la Comunicación interna se dedica a crear y mantener procesos de comunicación formativos e informativos con el trabajador. Investigación: La investigación se ha convertido en un elemento permanente, para generar mayor conocimiento de los públicos de interés. Se utilizan métodos y procedimientos que poseen rigor científico y a la vez facilitan la participación con los grupos implicados. Los estudios realizados contribuyen en gran medida al conocimiento de diversos tipos de innovación y experimentación de nuevas prácticas comunicativas. Técnicas de investigación utilizadas: Cualitativo comunicación interna y externa; comunicación interna(sondeos de opinión al personal); relaciones públicas (sondeos de opinión al público externo).

La evaluación: de la práctica comunicacional es otro elemento permanente en el sector, particularmente cuando se trata de evaluar o verificar la comprensión del mensaje en el público, y si, según los objetivos de comunicación, éste lo ha entendido como se esperaba que lo hiciera, pues dependiendo de ello, deberá tomarse una u otra decisión, esto 
es, continuar o ajustar la estrategia de comunicación. Se evalúa por fases: Especificación de objetivos, medición de objetivos, recogida y análisis de datos, comunicar resultados a quienes toman las decisiones; aplicación de resultados de la evaluación a las decisiones. Técnicas para evaluar la comunicación: evaluación y aceptación del mensaje; evaluación de política implementadas; auditoria de relaciones humanas; satisfacción de la comunicación, entre otros.

Sistemas utilizados para evaluar los resultados de las campañas de comunicación: midiendo el impacto en los medios; analizando el decremento/incremento de ventas; realizando auditorias de imagen; Participación de los clientes en acciones de comunicación; no se evalúan.

Dircom: En Colombia hace 10 años aproximadamente que esta figura viene tomando fuerza. Es la figura del profesional de la comunicación, que teniendo entre otras particulares, un gran poder de síntesis, de conciliación ante la diferencia, de una amplia fluidez mental, es a la vez un experto y hábil comunicador, totalmente integrado en la cultura corporativa de la organización a la que le sirve, y es quién depende directamente de la alta dirección, para coordinar, analizar, desarrollar, difundir y controlar la imagen pública de la misma. Por lo general los responsables de esta disciplina -Dircom- o directores de comunicación, son periodistas y/o comunicadores sociales de profesión, en formación creciente, participan de concursos de postgrados y Máster en su currículum, con alto conocimiento técnico de los productos y servicios de la empresa, creatividad y capacidad de trabajo en equipo. Este dato le da la confianza a la dirección general, de tener directores conscientes de su responsabilidad, con la formación y conocimientos necesarios para influir acertadamente en las decisiones de la empresa, y para coordinar, analizar, desarrollar, difundir y controlar la imagen pública de la misma.

Son quienes asumen el liderazgo de las personas, han sido investidas con el poder de dirigir, de integrar, movilizar, y de consolidar redes internas, de canales de difusión de información, de razonable amplitud y credibilidad. La situación del responsable de comunicación en el organigrama de la empresa nos demuestra la importancia que la función de Comunicación va adquiriendo en la organización moderna. La mayoría de los responsables de comunicación dependen directamente de la Presidencia, del Director General ó de la Secretaria General; de esta manera, por lo menos en la teoría, se aprecia el compromiso de la entidad con las propuestas realizadas por el equipo de comunicación y cierta autonomía en las decisiones importantes, condición sustancial para la aplicación de estrategias, para la selección de miembros del departamento o de agencias externas colaboradoras, y para influenciar la Dirección de la empresa.

Gestión situaciones especiales: situaciones de crisis, de emergencias y de negociación, donde la comunicación es un elemento esencial y requiere de un tratamiento especial, de atención, profesionalidad y responsabilidad en la Rep- 
utación Corporativa, en la Gestión de la Marca y la Responsabilidad Social Corporativa en las organizaciones.

Nuevas tecnologías: hay que destacar que los comunicadores utilizan de forma muy frecuente las redes sociales, el correo electrónico, el ordenador personal en el trabajo e Internet. También han aumentado en su frecuencia el uso la Intranet, utilizada de forma muy frecuente y el ordenador en casa. A través de la Web miles de empresas, organizaciones, otros medios de comunicación e individuos hablan de sí mismos, venden sus productos y promocionan sus ideas.

Consultoras de comunicación: La relación de las consultoras de comunicación con las empresa es muy alto, la frecuencia con la que se contacta las empresas con las consultoras de comunicación es por lo general a diario. La relación contractual empresasconsultoras son por: contrato formal; Presupuesto firmado; contrato verbal ó por carta de encargo. El departamentos que más contratan la consultaría de comunicación son: Comunicación; Marketing; Dirección General; presidencia; Relaciones Externas; Consejero Delegado; gabinete de prensa.

Los criterios de las empresas para contratar consultoras: Calidad del equipo de trabajo; Conocimiento del sector/especialización; Servicio al cliente; es una compañía de reconocido prestigio; creatividad; referencias; precios; estabilidad del estaff; capacidad de investigación; Relación personal; Presentación de credenciales.
Los factores que tienen mayor incidencia en el aumento del sector de comunicación y las relaciones publicas: mayor preocupación por la imagen corporativa; mayor credibilidad; saturación en los medios tradicionales; Relación coste/efectividad; actividad incipiente.

Los servicios de comunicación realizados por las empresas contratantes: Ejecución de eventos; Gabinete de prensa; Diseño pagina Web; Apoyo en situaciones de crisis; emplazamiento en ferias; Diseño gráfico; auditorias comunicación e imagen; Búsqueda de alianzas; búsqueda de ordenadores para oradores; Relación con los medios; Relaciones Institucionales; Reputación Corporativa; Comunicación de crisis; Identidad corporativa; Comunicación Interna; Relaciones con la Comunidad; Relaciones con el gobierno.

Finalmente podemos concluir que la Comunicación ha experimentado crecimiento y evolución, como ciencia no deja de conseguir nuevos hallazgos y nuevos retos, es una ciencia joven pero sin ninguna duda ya consolidada y dispuesta desde su propio armazón para dar muchos y profundos frutos a la sociedad. Las tendencias futuras del sector está dirigida a la especialización por sectores, especialización por servicios, servicios plenos, globalización, integración en grandes grupos de comunicación, integración en grandes grupos de publicidad. Es importante el modelo simétrico bidireccional en la gestión con todos sus públicos internos, externos. Ello conduce inevitablemente a una mayor eficacia de la organización, asegurando que las audiencias sean informadas y aten- 
didas de forma adecuada en cantidad y calidad, a través de estrategias con enfoque hacia el gerenciamiento corporativo con valiosas herramientas de gestión, como las políticas de comunicación e información que fortalecen la imagen de empresas sólidas, rentables, serias, confiables y con proyección futura.

\section{Referencias}

Alvarez A. (s.f.) "Medición y evaluación en Comunicación".

Arceov, J. L. (1999). Tratado de publicidad y relaciones publicas, Madrid: ICIE, Universidad Complutense de Madrid.

Arceo, J. L. (2003), Perfil de los profesionales de la Comunicación Persuasiva en España, Madrid: Instituto de Comunicación Institucional y Empresarial de la Universidad Complutense de Madrid.

Arrollo, L. (2003), Los cien errores de la comunicación en las organizaciones: ideas, casos y consejos para la excelencia en las relaciones públicas, Madrid: ESIC.

Capriotti, P. (2009), Branding corpo- rativo.

Costa, J. (2006), Imagen corporativa en el siglo XXI, Buenos Aires: La Crujía.

Costa, J. (1995), Comunicación Corporativa, Ediciones de las ciencias sociales, Madrid.

Costa ,J. (2004), La comunicación en acción: "informe sobre la nueva cultura de la gestión", Barcelona: Paidós.

Costa, J. (1977), "La imagen de empresa-métodos de comunicación integral", Biblioteca de la comunicación del CIAC, Barcelona.

Fajardo Valencia \& Nivia, A. M. (2016), "Relaciones Publicas y Comunicación Organizacional".

Fernández del Moral, J. \& Estever, F. (1999), "Áreas de especialización periodística”, Madrid: Fragua.

Fernandez Collado, C. (2002), La comunicación en las organizaciones, México: Trillas.

Grunig, J. E., Todd H. (2003), Dirección de Relaciones Públicas, Barcelona: Gestión, Edición adaptada por Jordi Cifra. 
Graciela Tristancho Cediel

106 | PolianteA | p. 106-106 | VOLUMen XIII | NÚMero 24 | AgOSTO 2017 